\title{
Cyclotomy and Ramanujan Sums in Quantum Phase Locking
}

\author{
Michel Planat ${ }^{\dagger 1}$ and Haret C. Rosu ${ }^{\ddagger 2}$ \\ ${ }^{\dagger}$ Laboratoire de Physique et Métrologie des Oscillateurs du CNRS, 25044 Besançon Cedex, France \\ ${ }^{\ddagger}$ Applied Mathematics, IPICyT, Apdo. Postal 3-74 Tangamanga, San Luis Potosí, México
}

\begin{abstract}
Phase-locking governs the phase noise in classical clocks through effects described in precise mathematical terms. We seek here a quantum counterpart of these effects by working in a finite Hilbert space. We use a coprimality condition to define phase-locked quantum states and the corresponding Pegg-Barnett type phase operator. Cyclotomic symmetries in matrix elements are revealed and related to Ramanujan sums in the theory of prime numbers. The employed mathematical procedures also emphasize the isomorphism between algebraic number theory and the theory of quantum entanglement.
\end{abstract}

Time and phase are amongst the most federal concepts of science. Hersh mentions Kant as putting time before number and making the intuition of time the origin of arithmetics [1].

The present work can be considered as an extension of a longstanding effort to model phase noise and phase-locking effects that are found in highly stable classical oscillators. It was unambiguously demonstrated that the observed variability (i.e., the $1 / \mathrm{f}$ frequency noise of such oscillators) is related to the finite dynamics of states during the measurement process and to the precise filtering rules that involve continued fraction expansions, prime number decomposition, and hyperbolic geometry [2]-[4]. We are interested here in possible quantum counterparts of these effects. The problem of defining quantum phase operators was initiated by Dirac in 1927 [5]. After more than three quarters of a century, it is still an interesting and essentially open problem. For an excellent review, see [6]. Our starting point is the Pegg and Barnett (PB) quantum phase formalism [7]-[8] where the calculations are performed in a Hilbert space $H_{q}$ of finite dimension $q[9]$. In the $\mathrm{PB}$ framework the phase states are the so-called discrete quantum Fourier transforms (QFT) of the number states, i.e., the following superpositions

$$
\left|\theta_{p}\right\rangle=q^{-1 / 2} \sum_{n=0}^{q-1} \exp \left(\frac{2 i \pi p n}{q}\right)|n\rangle .
$$

The states $\left|\theta_{p}\right\rangle$ form an orthonormal set and in addition the projector over the subspace of phase states is $\sum_{p=0}^{q-1}\left|\theta_{p}\right\rangle\left\langle\theta_{p}\right|=1_{q}$ where $1_{q}$ is the identity operator in $H_{q}$. The inverse QFT implies $|n\rangle=q^{-1 / 2} \sum_{p=0}^{q-1} \exp \left(-\frac{2 i \pi p n}{q}\right)\left|\theta_{p}\right\rangle$. As the set of number states $|n\rangle$, the set of phase states $\left|\theta_{p}\right\rangle$ is a complete set spanning $H_{q}$. In addition, the QFT operator is a $q \times q$ unitary matrix of entries $\kappa_{p n}^{(q)}=\frac{1}{\sqrt{q}} \exp \left(2 i \pi \frac{p n}{q}\right)$.

From now on, we restrict ourselves to the class of phase states $\left|\theta_{p}^{\prime}\right\rangle$ for which $p$ and $q$ satisfy the coprimality condition $(p, q)=1$, where $(p, q)$ is the greatest common divisor of $p$ and $q$. Differently from the phase states $(1),\left|\theta_{p}^{\prime}\right\rangle$ form an orthonormal base of a Hilbert space whose dimension is given by the number of irreducible fractions $p / q$. This number is known in Mathematics as the Euler totient function $\phi(q)$.

\footnotetext{
${ }^{1}$ e-mail: planat@lpmo.edu; Fax: 33(0)381853998

${ }^{2}$ e-mail: hcr@ipicyt.edu.mx; Fax: 524448335412
} 
Guided by the analogy with the classical situation [2] we call the irreducible states the phase-locked quantum states. In the space of quantum states, they generate a cyclotomic lattice $L$ [10]-[14] with a generator matrix $M$ of matrix elements $\kappa_{p n}^{\prime(q)}$ and $(p, q)=1$ and size $\phi(q) \times \phi(q)$. We notice that the corresponding Gram matrix of $L$, namely $H=M^{\dagger} M$, has matrix elements $h_{n, l}^{(q)}=c_{q}(n-l)$, which are Ramanujan sums

$$
c_{q}(n)=\sum_{\substack{p=1 \\(p, q)=1}}^{q} \exp \left(2 i \pi \frac{p}{q} n\right)=\frac{\mu(r) \phi(q)}{\phi(r)}
$$

where $r=q /(q, n)$. Ramanujan sums are thus defined as sums over the primitive characters $\exp \left(2 i \pi \frac{p n}{q}\right),(p, q)=1$, of the group $Z_{q}=Z / q Z$. In the equation above, $\mu(r)$ is the Möbius function which is 0 if the prime number decomposition of $r$ contains a square, 1 if $r=1$, and $(-1)^{k}$ if $r$ is the product of $k$ distinct primes [15]. Ramanujan sums are relative integers that are quasiperiodic in $n$ with quasi period $\phi(q)$ and aperiodic in $q$ with a type of variability imposed by the Möbius function (introduced by Ramanujan in the context of the Goldbach conjecture). Recently, we demonstrated that Ramanujan's sums are also useful in the context of signal processing as an arithmetical alternative to the discrete classical Fourier transform $[16]^{3}$.

The projection operator over the subset of phase-locked quantum states $\left|\theta_{p}^{\prime}\right\rangle$ is calculated as the coprimality-constrained sum of one-state projectors, i.e.,

$$
P_{q}^{\text {lock }}=\sum_{\substack{p=0 \\(p, q)=1}}^{q-1} P_{p}^{\prime}=\sum_{\substack{p=0 \\(p, q)=1}}^{q-1}\left|\theta_{p}^{\prime}\right\rangle\left\langle\theta_{p}^{\prime}\left|=\frac{1}{q} \sum_{n, l=0}^{q-1} c_{q}(n-l)\right| n\right\rangle\langle l|,
$$

so that the matrix element of the projection is such that $q\left\langle n\left|P_{q}\right| l\right\rangle=c_{q}(n-l)$. This is similar to the elements of the cyclotomic Gram matrix and sheds light on the equivalence between cyclotomic lattices of algebraic number theory and the quantum theory of phase-locked states. The quantum phase-locking operator is defined

$$
\Theta_{q}^{\text {lock }}=\sum_{\substack{p=0 \\(p, q)=1}}^{q-1} \theta_{p}\left|\theta_{p}^{\prime}\right\rangle\left\langle\theta_{p}^{\prime}\right|=\pi P_{q}, \quad \text { where } \theta_{p}=2 \pi \frac{p}{q} .
$$

The Pegg and Barnett phase operator $\Theta_{q}[7]-[8]$ results from the removal of the coprimality condition $(p, q)=1$. Using the number operator $N_{q}=\sum_{n=0}^{q-1} n|n\rangle\langle n|$ a generalization of Dirac's commutator $\left[\Theta_{q}, N_{q}\right]=-i$ is obtained.

In contrast, the phase number commutator for phase-locked states as calculated from (4) is

$$
C_{q}^{\text {lock }}=\left[\Theta_{q}^{\text {lock }}, N_{q}\right]=\frac{\pi}{q} \sum_{n, l=0}^{q-1}(l-n) c_{q}(n-l)|n\rangle\langle l|,
$$

\footnotetext{
${ }^{3}$ In the discrete Fourier transform the signal processing is performed by using all roots of unity of the form $\exp (2 i \pi p / q)$ with $p$ from 1 to $q$ and taking their $n^{\text {th }}$ power $e_{p}(n)$ as basis function. We generalized the classical Fourier analysis by using Ramanujan sums $c_{q}(n)$ as in (2) instead of $e_{p}(n)$. This type of signal processing is more appropriate for arithmetical functions than is the ordinary discrete Fourier transform, while still preserving the metric and orthogonal properties of the latter. Notable results relating arithmetical functions to each other can be obtained using Ramanujan sums expansions while the discrete Fourier transform would lead instead to low frequency tails in the power spectrum.
} 
and has antisymmetric matrix elements $\left\langle l\left|C_{q}^{\text {lock }}\right| n\right\rangle=\frac{\pi}{q}(l-n) c_{q}(n-l)$.

The finite and cyclotomic quantum mechanical rules are mostly encoded in the expectation values of the phase operator and phase variance (see Figures 1-3).

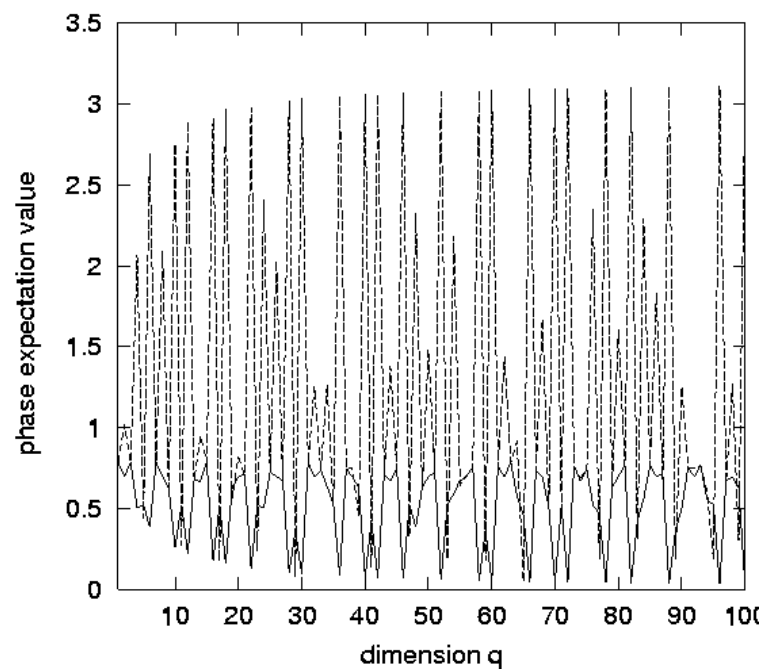

Fig. 1

The phase expectation value versus the dimension $q$ of the Hilbert space. Plain lines: $\beta=0$. Dotted lines: $\beta=1$

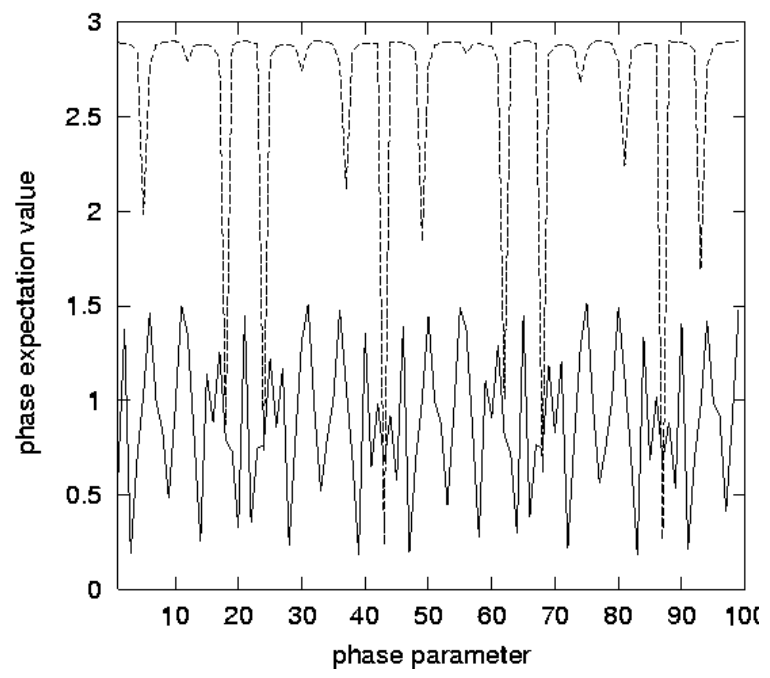

Fig. 2

The phase expectation value versus the phase parameter $\beta$. Plain lines: $q=15$. Dotted lines: $q=13$. 


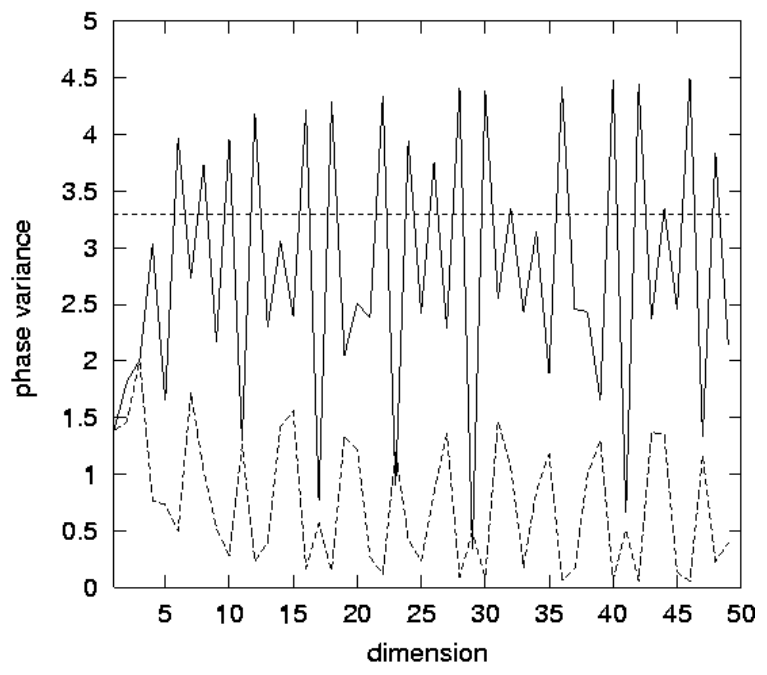

Fig. 3

The phase variance versus the dimension q of the Hilbert space. Plain lines: $\beta=1$. Dotted lines: $\beta=\pi$. The horizontal line at $\pi^{2} / 3$ is the variance of the classical phase.

Rephrasing Pegg and Barnett, we consider a pure phase state $|f\rangle=\sum_{n=0}^{q-1} u_{n}|n\rangle$ having $u_{n}$ of the form

$$
u_{n}=\frac{1}{\sqrt{q}} \exp (i n \beta)
$$

where $\beta$ is a real phase parameter. Defining the phase probability distribution as $\left\langle\theta_{p}^{\prime} \mid f\right\rangle^{2}$; the phase expectation value as $\left\langle\Theta_{q}^{\text {lock }}\right\rangle=\sum_{p} \theta_{p}\left\langle\theta_{p}^{\prime} \mid f\right\rangle^{2}$; and the phase variance as $\left(\Delta \Theta_{q}^{2}\right)^{\text {lock }}=$ $\sum_{p}\left(\theta_{p}-\left\langle\Theta_{q}^{\text {lock }}\right\rangle\right)^{2}\left\langle\theta_{p}^{\prime} \mid f\right\rangle^{2}$, where the summations are taken from $p=0$ to $q-1$ and $(p, q)=1$; the following result is obtained

$$
\begin{gathered}
\left\langle\Theta_{q}^{\text {lock }}\right\rangle=\frac{\pi}{q^{2}} \sum_{n, l=0}^{q-1} c_{q}(l-n) \exp [i \beta(n-l)], \\
\left(\Delta \Theta_{q}^{2}\right)^{\text {lock }}=4\left\langle\tilde{\Theta}_{q}^{\text {lock }}\right\rangle+\frac{\left\langle\Theta_{q}^{\text {lock }}\right\rangle}{\pi}\left(\left\langle\Theta_{q}^{\text {lock }}\right\rangle-2 \pi\right),
\end{gathered}
$$

with the modified expectation value

$$
\left\langle\tilde{\Theta}_{q}^{\text {lock }}\right\rangle=\frac{\pi}{q^{2}} \sum_{n, l=0}^{q-1} \tilde{c}_{q}(l-n) \exp [i \beta(n-l)],
$$

and the modified Ramanujan sums

$$
\tilde{c}_{q}(m)=\sum_{\substack{p=1 \\(p, q)=1}}^{q}(p / q)^{2} \exp \left(2 i \pi m \frac{p}{q}\right) .
$$

Fig. 1 illustrates the phase expectation value versus the dimension $q$ for two different values of the phase parameter $\beta$. For $\beta=1$, there are peaks at dimensions $q=p^{r}$, which are powers of a prime number $p$. For $\beta=0$, the peaks are smoothed out due to the averaging over the 
Ramanujan sums. Fig. 2 shows the phase expectation value versus the phase parameter $\beta$ for two dimensions of the Hilbert space, $q=15$ and $q=13$, respectively. For the case of the prime number $q=13$, the phase expectation value is of considerable higher level than for $q=15$, with absorption-like lines at isolated values of $\beta$. For $q=15$, which is not a prime power, the phase expectation value is at a much lower level and more random than for the case of the neighbour prime number.

Fig. 3 illustrates the phase variance versus the dimension $q$. The case of $\beta=1$ also leads to peaks at prime powers. Similar to the expectation value in Fig. 1, this case is thus reminiscent of the Mangoldt function $\Lambda(n)$ defined as $\ln p$ if $n$ is the power of a prime number $p$ and 0 otherwise. This function arises in the frame of prime number theory [2] in the formula for the logarithmic derivative of the Riemann zeta function $-\frac{\zeta^{\prime}(s)}{\zeta(s)}=\sum_{n=0}^{\infty} \frac{\Lambda(n)}{n^{s}}$. Its mean value oscillates around 1 with an error term which is explicitely related to the positions of Riemann zeros of $\zeta(s)$ on the critical line $s=\frac{1}{2}$. The error term shows a power spectral density close to that of $1 / f$ noise [2]. Finally, the phase variance is considerably smoothed out for $\beta=\pi$ and is much lower than the classical limit $\pi^{2} / 3$. Thus, the parameter $\beta$ is useful in defining quantum phase-locked states with small phase variances for a whole range of dimensions.

In conclusion, several interesting properties are pinpointed here as a result of introducing cyclotomic discreteness in the Pegg-Barnett quantum phase formalism. The interplay between results associated to prime number theory and quantum phase-locking phenomena looks quite stimulating. We recall that the idea of quantum teleportation was initially formulated by Bennett et al. in finite-dimensional Hilbert space [17]. Yet independently of this, one can conjecture that cyclotomic locking could play an important role in many fundamental tests of quantum mechanics related to quantum entanglement. As a matter of fact, Munro and Milburn [18] have already conjectured that the best way to see the quantum nature of correlations in entangled states is through the measurement of the observable, canonically conjugate of the photon number, i.e., the quantum phase. Moreover, in their paper dealing with the Greenberger-Horne-Zeilinger quantum correlations, Munro and Milburn presented a homodyne scheme requiring discrete phase measurement, yet to be experimentally realized in the ultrahigh detector efficiency limit. Nevertheless, a homodyne scheme automatically implies phase locking measurements with cyclotomy playing a role. We also recall that a universal algorithm for the optimal quantum state estimation of an arbitrary finite dimensional system in terms of a sequence of projectors has been introduced by Derka et al [19]. In one of their two examples, the sequence of projectors is very similar to the Pegg-Barnett projectors (in fact they noticed the analogy with the PB phase operator). Therefore, the quality of the cyclotomic phase estimation through a direct generalization of the maximal mean fidelity introduced in their paper could be utilized as well. It may be possible that the type of quantum phase-locking described in this paper may have some applications to the synchronization of remote oscillators by quantum entanglement as already proposed in [20].

\section{References}

[1] R. Hersh, What is Mathematics Really ?, Oxford Univ. Press, 1997.

[2] M. Planat, Fluct. \& Noise Lett. 1 (2001) R65.

[3] M. Planat and E. Henry, Appl. Phys. Lett. 80 (2002) 13. 
[4] M. Planat, "Modular functions and Ramanujan sums for the analysis of $1 / \mathrm{f}$ noise in electronic circuits", hep-th/0209243.

[5] P.A.M. Dirac, Proc. Roy. Soc. London A 114 (1927) 243.

[6] R. Lynch, Phys. Rep. 256 (1995) 367.

[7] D.T. Pegg and S.M. Barnett, Phys. Rev. A 39 (1989) 1665.

[8] D.T. Pegg and S.M. Barnett, J. Mod. Opt. 44 (1997) 225.

[9] There is a vast literature on finite quantum systems and number-theoretic results in this context. See, e.g., A. Vourdas and C. Bendjaballah, Phys. Rev. A 47 (1993) 3523.

[10] M. Craig, Mathematika 25 (1978) 44.

[11] J.H. Conway and N.J.A. Sloane, Sphere Packings, Lattices and Groups, Springer, New York, 1999, p. 227.

[12] E. Bayer-Fluckiger, Contemp. Math. 241 (1999) 69.

[13] J.B. Bost and A. Connes, Selecta Mathematica, New Series 1 (1995) 411.

[14] P. Cohen, J. de Théorie des Nombres de Bordeaux 11 (1999) 15.

[15] G.H. Hardy and E.M. Wright, An Introduction to the Theory of Numbers, Oxford Press, Oxford, 1979, p. 237.

[16] M. Planat, H. Rosu, S. Perrine, Phys. Rev. E 66 (2002) 56128.

[17] C.H. Bennett et al., Phys. Rev. Lett. 70 (1993) 1895.

[18] W.J. Munro and G.J. Milburn, Phys. Rev. Lett. 81 (1998) 4285.

[19] R. Derka, V. Bužek, A.K. Ekert, Phys. Rev. Lett. 80 (1998) 1571.

[20] M.S. Shariar, "Frequency locking via phase mapping of remote clocks using quantum entanglement", quant-ph/0209064. 